\title{
Posterior communicating artery aneurysm presenting with haemorrhage into an arachnoid cyst
}

\author{
R A Barker, R R Phillips, I F Moseley , W J Taylor, N D Kitchen, J W Scadding
}

\begin{abstract}
A patient is reported on with a subarachnoid haemorrhage (SAH) from an aneurysm of the posterior communicating artery, who initially presented with a sentinel bleed into an arachnoid cyst and normal magnetic resonance angiography (MRA) of the intracranial vasculature which led to a delay in diagnosis. Although this is a very rare presentation of a relatively common condition, it is important to recognise the importance of intracystic haemorrhage in such circumstances as well as the limitations of MRA, as a delay in diagnosis may have serious clinical consequences.

(F Neurol Neurosurg Psychiatry 1998;64:558-560)
\end{abstract}

Keywords: subarachnoid haemorrhage: arachnoid cyst: MRA: angiography

Subarachnoid haemorrhage (SAH) presents classically with the sudden onset of severe headache associated with nausea, vomiting, photophobia, and meningism. Often the patient loses consciousness, and the diagnosis is apparent clinically and confirmed by appropriate imaging of the brain or the finding of blood in the CSF. ${ }^{1}$ However, on occasions the diagnosis can be difficult to make especially when the history is less clear cut, ${ }^{1}$ and the combination of CT and MRI is essential to show the presence of acute haemorrhage, as MRI in isolation can be misleading. ${ }^{2}$ We present a case in which the diagnosis of an SAH secondary to an intracranial aneurysm was delayed because despite the abnormal CT and MRI, the MRA of the intracranial vessels was normal and the predominant bleed was into an arachnoid cyst with only small amounts of blood seen at other subarachnoid sites.

\section{Case history}

A 29 year old woman in the 28th week of her third pregnancy presented to the antenatal ward with an abrupt onset of severe generalised headache, nausea, vomiting, photophobia, and neck stiffness. Examination by a neurologist disclosed no focal neurological deficit and minimal neck stiffness. Brain CT disclosed a trace of high attenuation consistent with subarachnoid blood surrounding the medial aspect of the left temporal lobe with extension into the sylvian fissure. In addition there was a paucity of CSF around the basal cisterns with a degree of hydrocephalus, suggesting possible early obstructive hydrocephalus secondary to a recent subarachnoid haemorrhage. She had further imaging with MRI at this time, and this disclosed blood within an arachnoid cyst in the anterior part of the left middle cranial fossa (fig A, B), as well as confirming the degree of ventricular dilatation seen on CT. Analysis of CSF was not undertaken at this stage, partly because the diagnosis was made on imaging but more importantly because of the degree of hydrocephalus. MRA of the intracranial vasculature was then undertaken and was normal, with no evidence of either vessel spasm or an aneurysm. The patient's symptoms settled with conservative treatment and a diagnosis was made of a subarachnoid haemorrhage into an arachnoid cyst, possibly traumatic in origin, but with no evidence for an underlying aneurysm. Tests for a haemorrhagic diathesis were normal.

Her headache recurred six weeks later as the patient was entering the 35th week of pregnancy. On this occasion, the pain was felt more around the left eye and was again associated with vomiting but only mild photophobia and no consistent neck stiffness. MRI was unchanged, with no evidence of any fresh haemorrhage. Her headache worsened 48 hours later and was now clearly associated with neck stiffness and a low grade pyrexia $\left(37.4^{\circ}\right)$. The MRI, apart from showing some organisation of the blood products in the arachnoid cyst, was otherwise unchanged from before (fig C). However, 24 hours after the worsening of the headache, she developed a left sided ptosis with a partially dilated pupil that responded sluggishly to light. Examination of the CSF confirmed the diagnosis of a further SAH with over 50000 red blood cells $/ \mathrm{ml}$ in three successive samples, and carotid angiography confirmed the clinical diagnosis of an aneurysm of the left posterior communicating artery (fig D).

Thirty six hours later the patient underwent two combined operative procedures, to deliver the baby in a controlled manner (and avoid the 

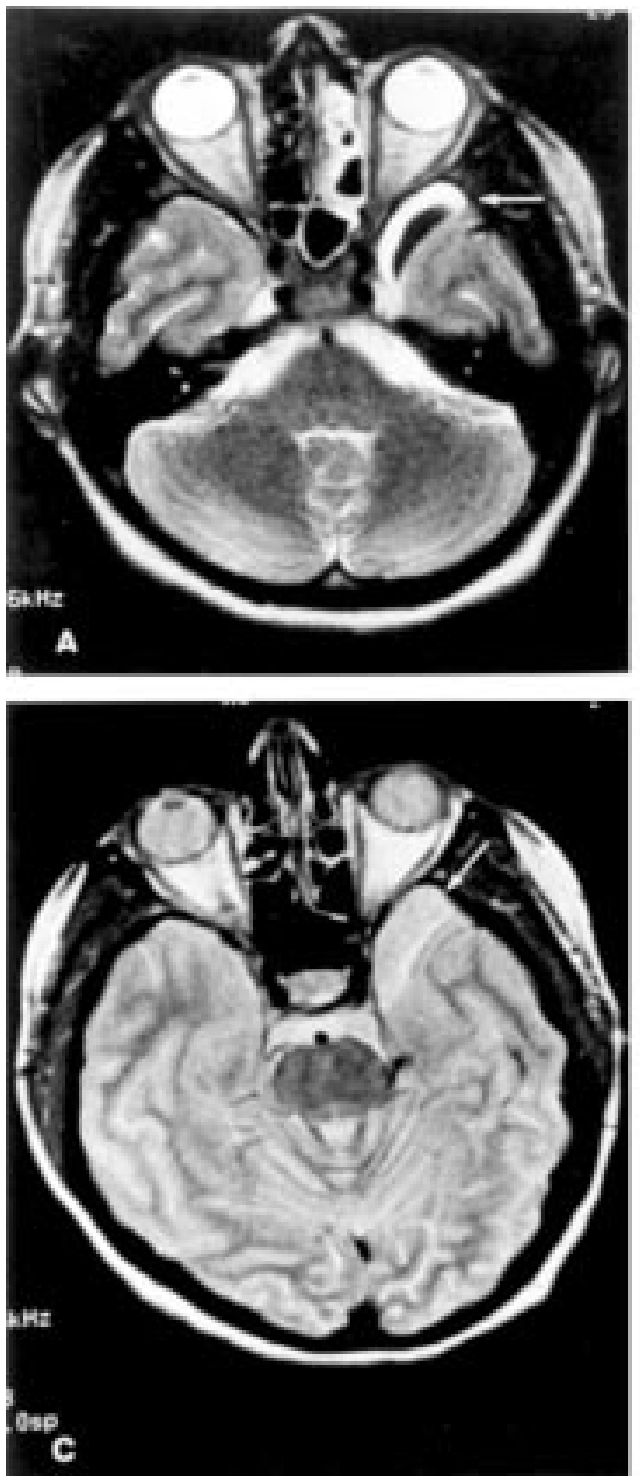

MRI and cerebral angiography. (A) Axial MRI showing blood within an arachnoid cyst at the tip of the left temporal lobe with a degree of ventricular dilatation. This is also obvious in the coronal plane (B). (C) MRI at the time of development of the partial left third nerve palsy shows organisation of the blood products within the cyst. (D) Intra-arterial angiography confirmed the presence of an aneurysm of the left posterior communicating artery.

high risk of precipitating premature labour in a multiparous patient close to term) and clip the aneurysm. Firstly, a live male baby was delivered by a lower section caesarian section under epidural anaesthesia, then the aneurysm was clipped in the usual way under general anaesthesia via a craniotomy. At operation, fresh blood was identified in the subarachnoid space with evidence of old haemorrhage within an arachnoid cyst. Both the patient and her baby boy made a full and uneventful recovery.

\section{Discussion}

Arachnoid cysts in the middle cranial fossa are common, and are being recognised in increasing numbers of patients as a result of improved imaging with CT and MRI. These cysts, which are thought to be congenital in nature secondary to arachnoid maldevelopment, very rarely cause problems, and when they do it is usually as a result of local pressure from the cyst on neighbouring structures. ${ }^{3}$ They are however,
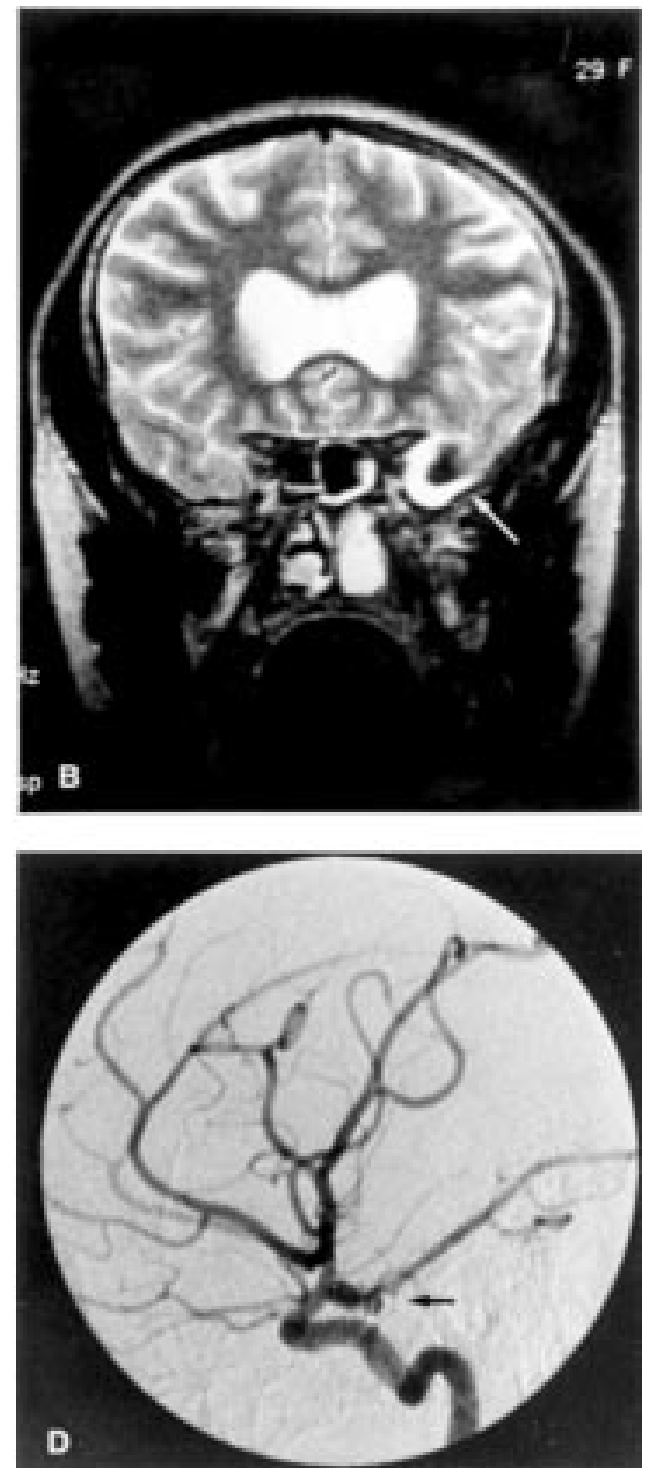

associated with subdural haematomas, possibly because of the fluid dynamics within the cyst and adjacent brain tissue and the shearing forces that are generated in response to minor head trauma. ${ }^{4}$ In such cases bleeding is often confined to the subdural space, and may, indeed be uniquely contralateral, but intracystic haemorrhage has only rarely been described, and is probably secondary to rupture of intracystic or bridging vessels. ${ }^{5}$ However, the association of leaking intracerebral aneurysms and blood within arachnoid cysts has only rarely been described. Hirose et al recently reported a patient with a classic history of SAH who on imaging had blood within an arachnoid cyst. ${ }^{6}$ Cerebral angiography disclosed an aneurysm at the bifurcation of the left internal carotid artery which was surrounded by the wall of the arachnoid cyst. Jinkins et al similarly described a patient in whom SAH from a middle cerebral bifurcation aneurysm was associated with blood within an 
arachnoid cyst. ${ }^{7}$ No cases with aneurysms of the posterior communicating artery have been described, despite the fact that most arachnoid cysts are in the anterior half of the middle cranial fossa. Furthermore, no cases of apparent spontaneous intracystic haemorrhage in the absence of an associated subdural haematoma have been described, which highlights the need for the clinician to search for an underlying aneurysm in cases such as the one we present.

The advent of better cranial imaging with MRI, although highlighting the relatively high incidence of arachnoid cysts and their benign nature, can, nevertheless, be misleading on occasions. In this case the $\mathrm{CT}$ at the original presentation was diagnostic of a recent SAH with early obstructive hydrocephalus, although the distribution of blood around the temporal lobe was a little unusual. Imaging with MRI at this time clearly showed blood in an arachnoid cyst but failed to show any other evidence for a subarachnoid bleed. This unusual MRI appearance coupled with the absence of meningitic signs and the normal MRA, misled us into diagnosing a spontaneous intracystic haemorrhage with no evidence of an underlying aneurysm. Indeed, even on analysing the reformatted MRA study retrospectively, there was no evidence of an aneurysm - so confirming that this was a real false negative result, and highlighting the limitations of this technique especially in the visualisation of aneurysms of the posterior communicating artery. Brain MRI is poor at documenting acute haemorrhage in the subarachnoid space unless the SAH is large, ${ }^{2}$ and in retrospect the diagnosis of spontaneous haemorrhage into an arachnoid cyst relied too heavily on the MRI and negative MRA rather than the CT findings. A point emphasised by the absence of changes (apart from some organisation of the blood products in the arachnoid cyst) on the MRI at her second presentation. However, on this occasion the headache was in association with focal neurological signs, and the diagnosis became obvious, despite the lack of change in the MRI.

We therefore present a case of SAH presenting with a sentinel bleed into an arachnoid cyst. The importance of this case is that haemorrhage into an arachnoid cyst with a history consistent with SAH should alert the clinician to the presence of an aneurysm. The use of MRI alone, or in conjunction with MRA, in such situations may be misleading and CT imaging with angiography are the more useful investigations.

1 Kase CS. Intracerebral hemorrhage. In: WG Bradley, RB Daroff, GM Fenichel, et al, eds. Neurology in clinical practice.
Vol 2: The neurological disorders. Boston: ButterworthHeinemann, 1996:1032-47

2 Ogawa T, Inugami A, Shimosegawa E, et al. Subarachnoid haemorrhage: evaluation with MR imaging. Radiology 1993;186:345-51

3 Shaw C-M, Alvord EC. "Congenital arachnoid" cysts and their differential diagnosis. Handbook of clinical neurology 1976;31:75-135.

4 Page A, Paxton RM, Mohan D. A reappraisal of the relationship between arachnoid cysts of the middle fossa and chronic subdural haematoma. F Neurol Neurosurg Psychiatry 1987;50:1001-7.

5 Eustace S, Toland J, Stack J. CT and MRI of arachnoid cyst with complicating intracystic and subdural haemorrhage. $\mathcal{F}$ Comput Assist Tomogr 1992;16:995-7.

6 Hirose S, Shimada S, Yamaguchi N, et al. Ruptured aneurysm associated with arachnoid cyst: intracystic hematoma without subarachnoid hemorrhage. Surg Neurol 1995;43:352-6.

7 Jinkins JR, Siqueira EB, Holoubi A. Ruptured middle cerebral aneurysm with accumulation of subarachnoid blood within convexity arachnoid cyst. Computerized Radiology 1987;11:185-7. 